\title{
Oberflächenprozesse - ein altes oder ein neues Thema?
}

\author{
Richard Dikau, Bonn
}

\section{Einleitung}

Der vorliegende Aufsatz beruht auf einigen Aspekten des Vortrages «Die Formung der Erdoberfläche - geomorphologische Perspektiven», der im Rahmen des Workshops «Erdoberflächenprozesse» gehalten wurde, der vom 25. bis 26. November 2005 in Göttingen stattgefunden hat. Die Beiträge und Diskussionen des Workshops bieten eine gute Gelegenheit, in diesem Themenheft der Geographica Helvetica einige grundsätzliche Aspekte geomorphologischer Zugänge zur Erdoberfläche sowie neuere Forschungsund Methodenfelder der Disziplin zu erörtern. Der Göttinger Workshop hatte Geowissenschaftler und Geomorphologen zusammengeführt, die diskutierten, in welchen Bereichen zukünftige gemeinsame Forschungsthemen weiterentwickelt werden können und möglicherweise programmatisch umzusetzen sind. Im Rahmen dieser Aktivitäten erscheint es angebracht, zunächst auf geomorphologische Paradigmen der «Erdoberfläche» einzugehen, da daraus Komplementaritäten zwischen den Disziplinen deutlich werden, die es mit Bedacht zu erkennen und zu würdigen gilt. Weiterhin sollen im Folgenden Aspekte der Theorie komplexer geomorphologischer Systeme angesprochen werden, um die es in der deutschsprachigen Disziplin merkwürdig still geblieben ist, um die allerdings in Nachbardisziplinen heftig gerungen wird.

\section{Geomorphologische Sichten auf die Erdoberfläche}

Für die Geomorphologie bilden die Erdoberflächenprozesse Komponenten der Formung, d.h. der Reliefformenentwicklung und -weiterbildung. Die Erdoberfläche selbst bildet ein geomorphologisches System mit folgenden Eigenschaften:

- 2-dimensionale Oberfläche eines 3-dimensionalen Körpers

- Oberfläche und Körper bilden die geomorphologische Form bzw. Reliefform

- Formung als Prozess oder Prozessgefüge der Materialabfuhr oder -zufuhr und damit des Formaufbaus und -abbaus

- Mehrphasig und polygenetisch

- Dualer Charakter der Reliefform (Variable der Prozesssteuerung und Produkt der Geomorphogenese)

- Größen-Existenzdauer-Relation.
Die Folgen von Mehrphasigkeit und Polygenetik zeigen sich in jedem zeitlichen Abschnitt der Formentwicklung in einer komplizierten räumlichen Struktur der Erdoberfläche. Unter geomorphogenetischen Gesichtspunkten werden die verschachtelten und hierarchisch strukturierten Reliefformen als Ausdruck unterschiedlich alter Formungsprozesse betrachtet und von BüDEL (1981) als Reliefgenerationen bezeichnet. Das Prinzip der Intensitätsauslese im Sinne RoHDEnBurgs (1989) bedeutet, dass in Aktivitätszeiten die Formung vorangetrieben wird, jedoch in Stabilitätszeiten Relikte der Aktivitätszeiten erhalten bleiben können, wenn die Effizienz der chronologisch jüngeren Prozesse schwächer ist. Reliefgenerationen sind mit dem Raum durch die Größe der Form und ihre Einbindung in das Gesamtrelief verknüpft. Diese räumliche Verschachtelung ist als Palimpsest beschrieben worden (CHORLEY et al. 1984), was bedeutet, dass ältere Formungsprozesse bereits abgeschlossen sind und als Vorzeitform noch im heutigen Georelief mehr oder weniger stark vorhanden sind. Sie bilden eine Reliefformenhierarchie. Das heutige Georelief kann somit ein mehr oder weniger stark entwickeltes Erbe bzw. Gedächtnis aus früheren Phasen der Formung enthalten, die dazu geführt haben, dass an einem Standort zeitlich nacheinander gebildete Formen angetroffen werden können, die ein räumlich verschachteltes polymorphes Reliefformenmuster darstellen, das bereits PENCK (1894) und PASSARGE (1912) beschrieben haben.

Derartige mehrphasig-polymorphe Muster bilden beispielsweise die deutschen Mittelgebirge, die aus einem verschachtelten System von Tälern, Talauen, Talterrassen, Hängen und Verflachungen bestehen (Kugler 1974; DiKau 1994), deren Bildung, Überformung sowie völlige oder teilweise Zerstörung in mehreren Formungsphasen bis in das Pleistozän und Tertiär zurückreichen, und die als Vorzeitformen bezeichnet werden (Bremer 1989). Mit diesen mesoskaligen Formengemeinschaften sind kleinere Formen verschachtelt, wie Erosionsgräben, Kolluvienkörper oder Flussterrassen, die einfacher aufgebaut sind (monomorph), jüngeren bzw. aktuellen Formungsphasen zugeordnet sind und als Jetztzeitformen bezeichnet werden. Dieser phänomenologische Zugang zum geomorphologischen System der Erdoberfläche wurde graphisch auf unterschiedlichste Weise dargestellt (z.B. BüdEL 1981; CHORLEY et al. 1984; Kugler \& Schaub 1997). Zur Illustration sei hier auf eine Darstellung von Denys Brunsden zurückgegriffen (BRUNSDEN 1996) (Abb.1). 


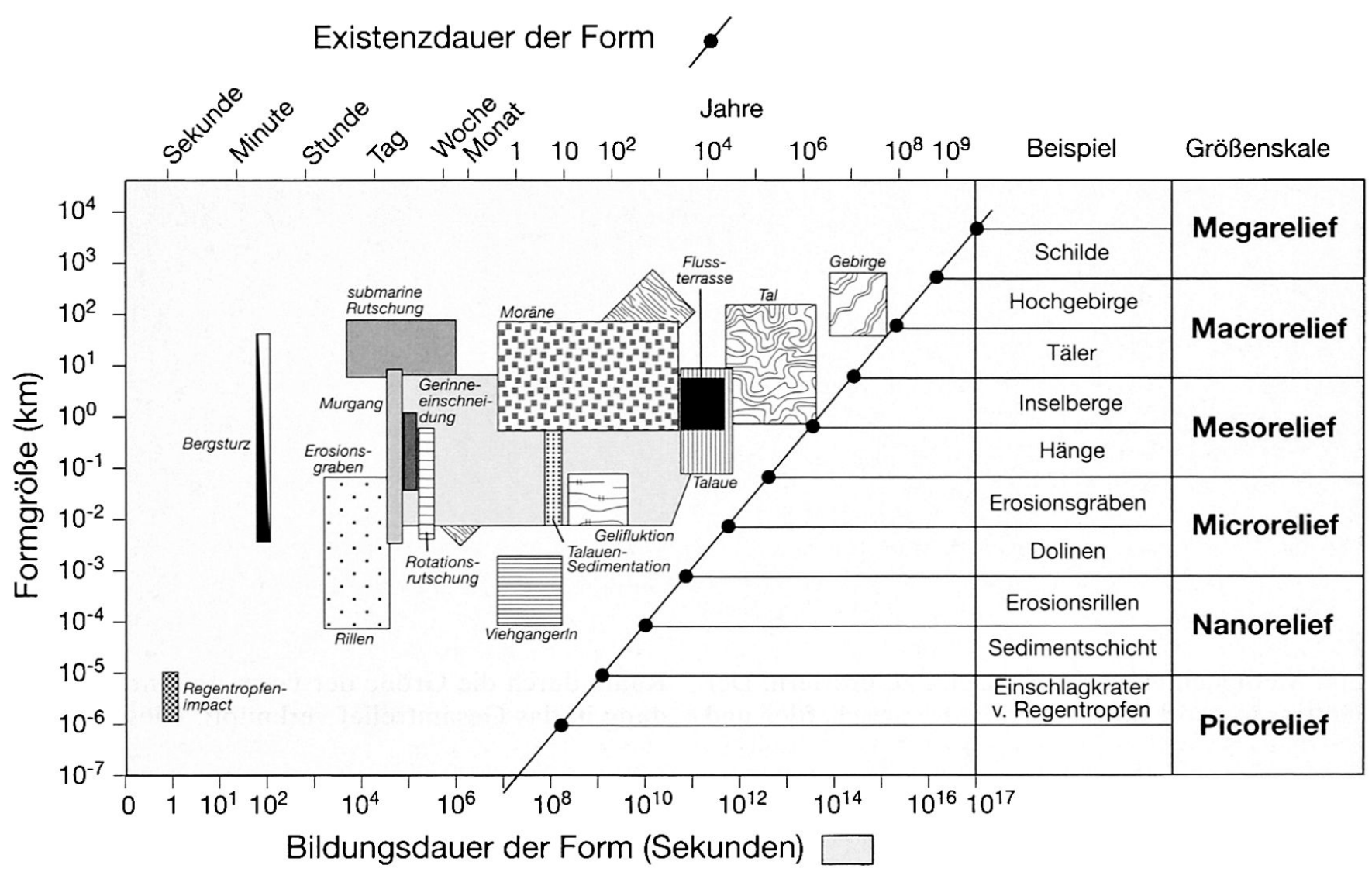

Abb. 1: Raum-Zeit-Skalen in geomorphologischen Systemen

Spatial and temporal scales in geomorphic systems

Echelles géographiques et temporelles dans les systèmes géomorphologiques

Quelle: BRUNSDEN 1996 (verändert)

\section{Methodische Konsequenzen der Multiskaligkeit geomorphologischer Systeme}

Die Frage, welche methodischen Konsequenzen aus dem multiskaligen Charakter geomorphologischer Systeme gezogen werden müssen, bildet seit mehreren Jahrzehnten einen zentralen Punkt der Diskussionen in der Disziplin. Es geht im Kern um die Frage, welche Zustände geomorphologische Systeme einnehmen können, und mit welchen Methoden Systeme in Modellen abgebildet werden können. Seit der Phase der paradigmatischen Ablösung der narrativ-historischen Ansätze der Geomorphogenetik (z.B. im Sinne von W.M. Davies, L. KING oder J. Büdel) durch die messende Prozessgeomorphologie in den 1950er Jahren fand in weiten Teilen der nationalen und internationalen Disziplin eine massive Verkleinerung der raumzeitlichen Skalen statt. Vor allem in den angloamerikanischen Ländern, jedoch zunehmend auch in anderen nationalen Gruppen, war der Preis dieser nomothetischen Wende die Aufgabe der großskaligen Geomorphogenetik regionaler, kontinentaler und globaler Ausdehnung. Die entstandene Dichotomie zwischen funktionalen und geomorphogenetischen Ansätzen würde jedoch dazu führen, so die Annahme zahlreicher Schulen, dass es in Zukunft möglich wäre, die kleinskalige Prozessbeschreibung mit der Geomorphogenese durch Methoden des analytischen Upscaling zu koppeln (vgl. die Diskussionen z.B. in MCCANN \& Ford 1996; RhOAds \& Thorn 1996; KirKby 1996; Beven 1996; Sugden 1996; Sugden et al. 1997; Bauer et al. 1999). Diese Behauptung blieb nicht unwidersprochen. Bereits in den 1970er Jahren forderte Sснumm (1991) skalenadäquate Methoden der funktionalen («modern») und der genetischen («historical») Raumzeit. KIRKBY (1999) hat diesen Ansatz weiterverfolgt. Er meint, dass die Analyse raumzeitlich geschachtelter Hierarchien geomorphologischer Systeme eine Konzeption geschachtelter Modelle erfordert (Abb. 2). Damit erfolgt eine Beschreibung der Formveränderung und der dafür verantwortlichen Prozesse am unteren Ende der Skalen durch kleinskalige Modelle mit stationären physikalischen Prozessbeschreibungen und am oberen Ende durch Massenbilanzansätze, die aus prozess-korrelaten Sedimentproxies z.B. mittlere Denudationsraten rekonstruieren. 


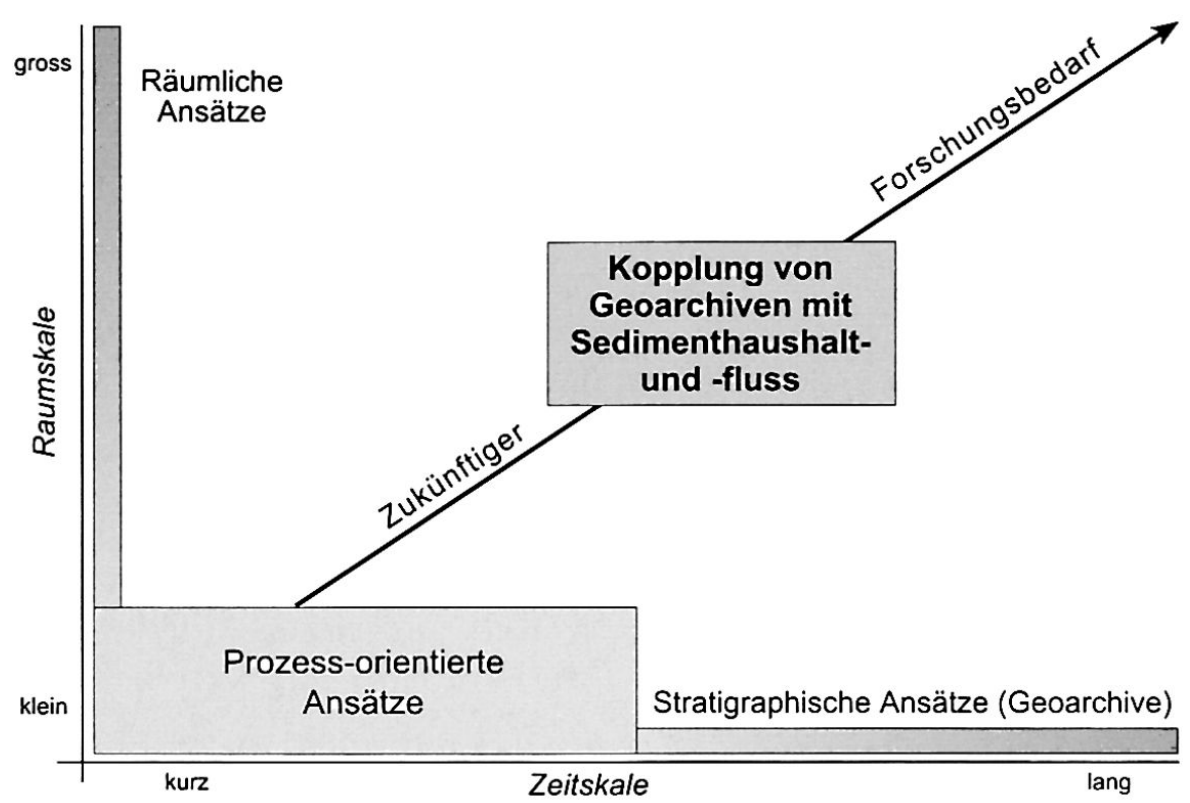

Abb. 2: Skalen heute bevorzugter stratigraphischer und raumbezogener Ansätze sowie des zukünftigen Forschungsbedarfes bei der Analyse von Flusssystemen

Scales of stratigraphic and spatial approaches and future research needs in fluvial system analysis

Echelles des approches stratigraphiques et spatiales et besoins de recherche futurs dans le domaine de l'analyse des systèmes fluviaux

Quelle: KIRKBY 1999 (verändert)

Eine weiterführende Problematik betrifft die Frage, in welcher Art und Weise formverändernde Prozesse kleinerer Skalen mit höherskaligen Phänomenen gekoppelt sind (Richards et al. 1995). Gibt es Rückkopplungen zwischen geomorphologischen Systemen unterschiedlicher Skalen oder können Reliefformen einer Skale, z.B. ein mesoskaliges Mittelgebirge, nur durch Prozesse der Mesoskale erklärt werden? Mit dieser Frage wird eine kritische Stellung zu den paradigmatischen Aussagen von Sснимм \& LichTY (1965) bezogen, die behaupten, dass zeitskalenspezifische Relationen zwischen den abhängigen und unabhängigen Variablen vorliegen. In dieser Arbeit bleibt darüber hinaus unklar, wie die Beziehung zwischen Skalen methodisch zu behandeln ist, wenn die Formungsprozesse der Altform nicht mehr auftreten und chronologisch folgende Formungsphasen anderen Prozessgefügen unterworfen sind. Damit verbunden ist die Frage, ob Reliefform und formender Prozess unterschiedlicher Raumzeit-Skalen einen selbstständigen Objektcharakter besitzen (DIKAU 1994).

\section{Kopplung endogener und exogener Prozesse}

Die Problematik der Kopplung funktionaler und geomorphogenetischer Konzeptionen hat in jüngster Zeit durch englische Geomorphologen neue Impulse erhalten. In mehreren Arbeiten hat Mike SummerFIELD zu Themen der Reliefentwicklung und der Kooperationspotenziale zwischen Geomorphologie und anderen geowissenschaftlichen Disziplinen Stellung bezogen (Summerfield 2000, 2005). In einem perspektivischen Aufsatz hat Summerfield (2005) darauf hingewiesen, dass die Geomorphologie durch geowissenschaftliche Nachbardisziplinen (z.B. Geologie, Geochemie und Geophysik) eine neue Bewertung und Würdigung erfahre. "Geomorphology matters", da zunehmend erkannt wird, dass die Oberfläche der Erde, also das geomorphologische Untersuchungsobjekt "per se», in neuen geophysikalischen Modellansätzen der Orogenese eine zentralere Rolle spielen sollte. Dies betrifft:

- die Verfügbarkeit globaler digitaler Höhendaten,

- thermochronologische Datierungstechniken sowie

- Datierungstechniken mit kosmogenen Nukliden.

Mit diesen Verfahren seien geomorphogenetische Modellierungen in längeren Zeitskalen mit weitaus verbesserter Plausibilität und Datengrundlage möglich. Dass die hohen Potenziale dieser Techniken, mit Ausnahmen, v.a. durch die Geophysik und Geochronologie und nicht durch die «geographische Geomorphologie» erkannt wurden, führt SUMmERFIELD (2005) auf die nach 1945 erfolgte Entwicklung der Geomorphologie hin zur Prozessgeomorphologie 
zurück. In der anglo-amerikanischen Disziplin sind dabei die Schwerpunkte auf die Prozessmodellierung gelegt worden mit massiver Einengung der Raumund Zeitskalen. Von daher war die "geographische Geomorphologie» ab Beginn der 1980er Jahre nur noch sehr schwach auf die wissenschaftlichen Potenziale der neuen Technologien der Altersdatierung und der geodynamisch-tektonischen Modellierung vorbereitet, in der Tektonik und Erdoberfläche ein gekoppeltes System darstellen. Nicht die (nomothetische) Entwicklung allgemeiner physikalischer Gesetze sei nun das Ziel geworden, sondern die (idiographische) Erklärung des Einzelfalles.

Welche geomorphologischen Fachkompetenzen, die in derartige Forschungsagenden eingebracht werden können, sieht Summerfield (2005) in der gegenwärtigen Phase?

- Ausgezeichnete geomorphologische Geländeerfahrung, die sinnhafte Variablen und Objekte für die numerische Modellierung der Reliefentwicklung liefert

- Kenntnisse und Modellierung geomorphologischer Phänomene, wie z.B. Glazialerosion oder durch tektonische Prozesse verursachte Verstärkung von Erosions- und Sedimentationsphänomenen

- Disziplingeschichtliche Kenntnisse zur Verhinderung der Neuerfindung des «geomorphologischen Rades»

- Aktive Bearbeitung der Schnittstelle zwischen den empirischen Befunden von Einzelprozessen und Prozessaggregationen, wie z.B. die Sedimenteintragspfade in fluviale Systeme oder die Integration der fluvialen Erosion in großskalige Modelle

- Verständnis der Schlüsselformelemente des geomorphologischen Systems, wie z.B. die HangGerinne-Kopplung, Knickpunktmigration in Gerinnen, Schichtstufenhänge oder fluviale Erosion im Festgestein.

Themenstellungen dieser Art erfordern eine Reihe von geochronologischen Techniken, die für die numerische Modellierung der Reliefentwicklung erforderliche Daten liefern und genutzt werden müssen. Es sei nun nicht mehr nötig, unhaltbare und systemisch falsche raum-zeitliche Extrapolationen von klein/kurzskaligen Messungen in die hohen Skalen zu versuchen. Nach Summerfield (2005) können diese Ansätze nur in multidisziplinären Forschungsteams verfolgt werden, die Geomorphologen, Geologen, Geophysiker und Geochemiker einschließen müssen.

\section{Komplexität geomorphologischer Systeme}

Bereits Stanley Schumm beschäftigte sich mit dem Problem komplexen Systemverhaltens in der Geo- morphologie (Sсhumm 1973, 1979; vgl. Dikau 2005). Die komplexe Reaktion («complex response») eines fluvialen Systems entsteht dadurch, dass in einem bestimmten Zeitraum ein externer Einfluss (z.B. eine Ansenkung der Erosionsbasis) innerhalb des Systems zu räumlich variablen Reaktionen führen kann. Hangabtrag, Einschneidung und Deposition können im selben Zeitraum räumlich benachbart auftreten. Die Begründung für dieses Systemverhalten liegt in der räumlich variablen Konfiguration des Systems sowie in den Eigenschaften der Systemspeicher, die unterschiedliche interne Schwellenwerte (variable Sensitivität) aufweisen. Für die Erklärung externer klimatischer und gesellschaftlicher Einflüsse auf ein geomorphologisches System aus Proxydaten eines Geoarchives (z.B kolluviale oder fluviale Sedimente) hat die komplexe Systemreaktion bedeutende Konsequenzen, da kausale Ursache-Wirkungsbeziehungen nicht mehr ohne weiteres zur Erklärung des Phänomens herangezogen werden können. PHILIPS (2003) benennt mehrere phänomenologische Ursachen für nichtlineares, komplexes Systemverhalten («complex nonlinear dynamics», CND):

- Schwellenwerte («thresholds»)

- Masse- und Energiespeicher («storages»)

- Sättigung und Entleerung («saturation and depletion»)

- Selbstverstärkung durch positive Rückkopplung («self-reinforcing by positive feedback»)

- Abschwächung durch negative Rückkopplung ( «self-limitation by negative feedback»)

- Konkurrierende Wechselwirkungen («competitive relationships»)

- Mehrfache Formen der Anpassung («multiple modes of adjustment»)

- Selbstorganisation («self-organization»)

- Hysterese («hysteresis»).

Trotz dieser Bemühungen, die Theorie komplexer Systeme in der geomorphologischen Forschung anzuwenden, ist ihr Potenzial in der Disziplin bei weitem noch nicht ausreichend rezipiert worden. Dies mag auch mit den paradigmatischen Konsequenzen der Theorie zusammenhängen. Nur wenige Beispiele sollen dies erläutern (vgl. auch FAvis-MorTLOcK 2004).

Die Theorie der Selbstorganisation bedeutet, dass in komplexen Systemen fernab thermodynamischer Gleichgewichte sich wechselseitig bedingende Prozesse zu dissipativen Strukturen führen können. Diese Systeme sind offen, ihnen wird ständig Energie entzogen, sodass sie, um zu überleben, ständig Energie aus ihrer Umwelt aufnehmen müssen. Dissipative Systeme können ohne externe Lenkung neue Ordnungsstrukturen bilden (Stabilität von Nichtgleichgewichten), sie sind selbstorganisiert. Die Selbstorganisation kann auf bestimmten räumlichen Skalenniveaus ange- 
siedelt sein. Diese Theorie ist eng mit dem ChemieNobelpreisträger von 1977, ILYA PRIGogine, verbunden (Prigogine 1992, 1997). Aus evolutionären (zeitabhängigen) Systemen, die durch irreversible Prozesse gekennzeichnet sind, können demnach geordnete Strukturen (raumzeitliche Muster) entstehen. Sind geomorphologische Systeme selbstorganisiert und geomorphologische Formen dissipative Strukturen? SPEDDING (1997) stellt eine derartige Hypothese auf. Danach sind geomorphologische Formen raumzeitlich variable Systemstrukturen, die durch den Vorgang der Selbstorgansiation gebildet worden sind.

Eine weitere diskussionswürdige Eigenschaft komplexer Systeme ist der Emergentismus, dessen Definition allerdings nicht unumstritten ist (STEPHAN 1999). Als emergent bezeichnet man systemische Eigenschaften, die nicht aus den subskaligen Elementen des Systems determiniert werden können. In diesem Sinne können daher z.B. mesoskalige kolluviale und alluviale Sedimentspeicher oder Gerinnenetzwerke als Emergenzen subskaliger Prozesse und Objekte des Partikeltransportes aufgefasst werden. Derartige mesoskalige Systemaggregate können allerdings nicht aus einem Verständnis der Eigenschaften des subskaligen Partikeltransportes heraus interpretiert werden. Die emergierten höherskaligen Strukturen dürfen also nicht an den Merkmalen der subskaligen Ebene ansetzen. Stattdessen etablieren sich auf jeder Skale ständig neue Systemeigenschaften («emergent properties», WASSON 2002), die eine skalenbezogene Epistemologie erfordern, so dass Modelle des «upscaling» nicht angewendet werden können (vgl. auch HARRISON 2001).

Ein weiteres Merkmal komplexer Systeme bedeutet die zeitlich variable räumliche Konfiguration («configurational status»), die das Produkt irreversibeler Prozesse der Systemevolution (Geomorphogenese) ist. Unter geomorphologischen Gesichtspunkten wird die Systemkonfiguration aus Komponenten gebildet, die geomorphometrisch beschrieben werden können, wie z.B. Flussnetze, die Topologie von Sedimentkörpern als Quellen und Senken, Kiesbänke in Gerinnen, Toposequenzen oder Hangcatenen (LANE \& Richards 1997; Trimble 1999; Richards 2002). Bei der Entwicklung des Systems werden diese Komponenten räumlich verändert und bilden damit jeweils neue Rahmenbedingungen für das zeitlich nachfolgende Prozessgefüge. Sie beschreiben die Pfadabhängigkeit («path dependence») der Systementwicklung. Das System strebt nach diesem Ansatz nicht mehr einen Gleichgewichtszustand an, sondern entwickelt sich (evolviert) entlang von Trajektorien, die die Richtung der zeitlichen Systementwicklung bezeichnen. Im Grunde wird mit dieser Theorie das aktualistische Axiom der Geowissenschaften hinterfragt, das besagt, dass direkte Rückschlüsse von heutigen Formungsprozessen der
Erdoberfläche in die Vergangenheit möglich sind. Die Pfadabhängigkeit heisst somit, dass die Veränderung eines geomorphologischen Systems als Reaktion auf ein externes Ereignis (Einfluss von Klima und Gesellschaft) von der Geschichte des Systems («history matters") und damit von der jeweiligen Konfiguration, auf die das Ereignis trifft, abhängig ist. Die analytische Rekonstruktion einer derartigen Systemgenese wird damit erheblich erschwert, da die variable Raumkonfiguration für jeden Zeitschritt bzw. für jede Zeitscheibe rekonstruiert werden muss, was hohe Anforderungen an geeignete Proxydaten und an die rekonstruktive Modellierung stellt.

Mit diesen wenigen Beispielen soll deutlich gemacht werden, dass die Komplexitätstheorie für die geomorphologische Wissenschaft hohe Relevanz besitzt. Dass diese paradigmatische Weiterentwicklung erforderlich ist, d.h., dass den empirischen Anomalien (im Sinne von Thomas KunN) in einer "Geomorphologie des Gleichgewichtes» nicht mehr ausreichend begegnet werden kann, hat Phillips (1992) bereits Anfang der 1990er Jahre behauptet. Von paradigmatischer Bedeutung ist die Komplexitätstheorie auch deshalb, weil die Axiome des tradierten mechanistischen Weltbildes nicht mehr hinreichend sind, um das Verhalten komplexer Systeme verstehen zu können (MAINZER 2004). Weiterhin besitzt die Theorie des Komplexen im Rahmen der sozial-ökologischen Erforschung gekoppelter Natur-Gesellschafts-Systeme eine zentrale Bedeutung (BECKER \& JAHN 2006). Wenn die deutschsprachige Geomorphologie in dieser «Dritten Säule» der «Mensch-Umwelt-Forschung» einen verbindlichen wissenschaftlichen Diskurs mitgestalten will, müssen die Komplexitätstheorie und ihre empirischen Konsequenzen einen weit höheren Stellenwert erlangen.

\section{Sedimenthaushalt}

Eine gleichermaßen neuere Entwicklung zur Bearbeitung des Skalenproblems in der Geomorphologie betrifft die Sedimenthaushaltsmodellierung. Als Schüler von Richard Chorley geht Olav SlaymaKER (1997) sogar so weit, dem Sedimenthaushalt den Status eines disziplinkonvergenten Ansatzes zuzusprechen. Der in der internationalen Forschung etablierte Begriff des Sedimenthaushaltes («sediment budget») umfasst sämtliche Komponenten eines geomorphologischen Systems in Form von Quellen-Senken-Kopplungen sowie die Sedimentflussprozesse zwischen diesen Komponenten. Der Ansatz umfasst die folgenden Teilprozesse:

- Abtrag auf Hängen

- Temporäre Zwischenspeicherung (z.B. Kolluvien, Schwemm- und Murkegel, Sturzmaterial, Talauen) 
- Sedimenttransporte in die fluvialen Gerinne

- Remobilisierung des zwischengespeicherten Materials unterschiedlicher Speichertypen

- Sedimentaustrag aus den Einzugsgebieten.

In der deutschen Geomorphologie wurden Versuche der Sedimentbilanzierung bereits vor dreissig Jahren durch Dietrich Barsch für subarktische und alpine Systeme durchgeführt (BARSCH 1981; BARSCH \& CAINE 1984). Für ein mesoskaliges Tributär des Mississippi demonstrierte Trimble (zuletzt 1999) die raumzeitliche Variabilität der Sedimentspeicherung in Kolluvien, fluvialen Sedimenten und des zeitlich davon entkoppelten Sedimentaustrags. In dieser Untersuchung wurde bereits in den 1980er Jahren die Notwendigkeit der quantitativen, zeitabhängigen Beschreibung des Sedimenthaushalts fluvialer Systeme postuliert.

Die räumliche und zeitliche Multiskaligkeit des Sedimenthaushaltes von Flusssystemen (BROWN \& QUINE 1999) und die Transformation von Geoarchivdaten in Modelle der Sedimentbilanz und des Sedimentflusses sind ein aktuelles Forschungsfeld der Geomorphologie des Holozäns. Wie aus den Arbeiten von Richards (2002) oder Dearing \& Jones (2003) hervorgeht, müssen für große Raum- und lange Zeitskalen empirische Bilanzmodelle entwickelt werden. Dabei wird die Ableitung von Sedimentflüssen aus Proxydaten der Geoarchive (Sedimentvolumen, Depositionsraten, Sedimentaustragsverhältnisse) versucht, die auf stratigraphisch-chronometrischen $\mathrm{Be}$ funden der kolluvialen und alluvialen Speicher beruhen. Die mit wachsender Raumskale zunehmende Komplexität des fluvialen Systems wird durch die $\mathrm{Zu}$ - oder Abnahme der Kopplung verschiedener Speicherkomponenten und der skalenspezifischen Reaktionen des Systems auf externe und interne Variablen charakterisiert. Dieses Phänomen ist auch damit zu erklären, dass durch die Kaskadenstruktur der Speicher externe Variablen, wie z.B. die Landnutzungsänderung, mit zunehmender Raumskale ihren Einfluss verlieren und durch system-interne Prozesse der Materialumlagerung überdeckt werden können (Abb. 3).

Derartige Ansätze werden seit dem Jahre 2001 im Bündelprojekt RheinLUCIFS der Deutschen Forschungsgemeinschaft (DFG) bearbeitet, das sich der Bedeutung klimatischer, gesellschaftlicher und systeminterner Einflüsse auf das fluviale System des Rheins widmet (Zimmermann et al. 2004; Dikau et al. 2005; Herget \& Dikau 2005; Dix \& Kleefeld 2005; Hoffmann et al. 2006; Houben et al. 2006). Die methodische Herausforderung besteht hier in der Kopplung zwischen Sedimentarchiv, Sedimenthaushalt und Sedimentfluss mit den systemexternen und -internen Variablen, wobei die Nichtlinearitäten im Systemverhalten eine besondere Rolle spielen (Abb. 4). Diese Forschungen sind in internationale Programme des International Council for Science (ICSU) (GREgory et al. 2006) und von Past Global Changes (PAGES) (DEARING et al. 2006a, 2006b) eingebunden.

Die Konsequenzen dieser Aussage lassen sich dahingehend zusammenfassen, dass die geomorphologische Prozessforschung auf jedweder RaumZeit-Skale mit Reliefformen rückgekoppelt werden muss. Voraussetzung jeder geomorphologischen Systemanalyse ist daher eine Bestandsaufnahme und Modellierung der räumlich verteilten Systemkomponenten und ihrer zeitlichen Trajektorien in Form des 3-dimensionalen Reliefs und seiner subordinierten Reliefelemente. Interessant ist, dass die Würdigung von Form-Prozess-Form-Rückkopplungen in Prozess-Reaktions-Systemen und die Bedeutung der räumlichen Konfiguration von Reliefformen und ihrer zeitlichen Variabilität («configurational status») in Teilen der reduktionistisch geprägten englischen Geomorphologie zu einer Renaissance der Reliefform und ihrer Quantifizierung geführt haben (z.B. LANE \& Richards 1997). Kolluviale und alluviale Sedimentkörper bilden Strukturelemente dieser Systeme. Sie sind Komponenten des Georeliefs und geomorphometrisch quantifizierbar (DIKAU \& Schmidt 1999). Die Quantifizierung stützt sich auf multiskalige Ansätze für 2- und 3-dimensionale Objekte. Hier kann auf die geomorphometrischen Ansätze der deutschen Geomorphologie (KugLER 1974; RASEMANN 2004) zurückgegriffen werden. Zusammenfassend ergibt sich damit eine viel versprechende Kopplung von drei Ansätzen der Geomorphologie zu einer Forschungskonzeption, die als Basis für die Modellierung des Sedimenthaushaltes fluvialer Systeme genutzt werden kann.

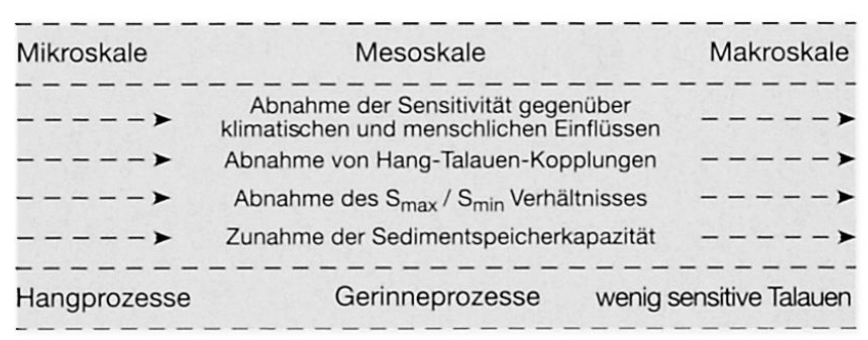

Abb. 3: Skalenabhängiger Variableneinfluss in fluvialen Sedimentkaskadensystemen

Scale-related influence of variables in fluvial sediment cascade systems

Influence des variables des systèmes fluviaux sédimentaires relativement aux différentes échelles 
Proxy-Parameter

\begin{tabular}{|c|c|c|c|}
\hline \multicolumn{2}{|c|}{ Sedimentdaten/-archiv (SA) } & \multicolumn{2}{|c|}{ Sedimentfluss (SF) } \\
\hline \multicolumn{4}{|c|}{ Sedimenthaushalt } \\
\hline \multirow{3}{*}{$\begin{array}{l}\text { Externe/ } \\
\text { interne } \\
\text { Variablen }\end{array}$} & Konfiguration & \multirow{3}{*}{$\begin{array}{l}\text { Nichtlinearität } \\
\text { Selbstorganisation } \\
\text { Emergenz }\end{array}$} & Haushalts- \\
\hline & Geomorphometrie & & \\
\hline & $\begin{array}{l}\text { v. Einzugsgebieten } \\
\text { Speichertyp } \\
\text { Gerinnenetz }\end{array}$ & & $\begin{array}{l}\text { Sedimentaustrag } \\
\text { Sedimentvolumen } \\
\text { Sedimentfracht } \\
\text { Depositionsrate }\end{array}$ \\
\hline
\end{tabular}

Abb. 4: Variablen und Komponenten in fluvialen Systemen, die für das Verständnis der Transformation des Sedimentarchivs in den Sedimentfluss von Bedeutung sind.

Variables and components in fluvial systems important for the understanding of sediment archive to sediment flux transformation.

Variables et composantes des systèmes fluviaux nécessaires à la compréhension des transformations affectant les dépôts et les flux de sédiments.

\section{Abschließende Bemerkungen}

Mit der Jahrhundertwende steht die geomorphologische Disziplin vor besonderen und einschneidenden Herausforderungen. Von zentralem Interesse erscheinen die Wechselwirkungen zwischen natürlichen und sozialen Systemen im Lichte einer sich verschärfenden Umweltkrise sowie veränderte paradigmatische Grundlagen der Systemtheorie. Dazu wurde in mehreren Aufsätzen Stellung bezogen und es wurden folgende Themenbereiche hervorgehoben (DIKAU 1999, 2004, 2005):

- Wissenschafts- und erkenntnistheoretischer Diskurs

- Geomorphologische Systemtheorie und ihre Weiterentwicklung

- Nichtlinearität und Komplexität geomorphologischer Systeme

- Skalenabhängige Konfiguration des geomorphologischen Raumes

- Sedimenthaushalt und -fluss in biogeochemischen Systemen

- Naturgefahren und -risiken.

Von übergeordneter Bedeutung scheinen die Aufgaben in den inter- und transdisziplinären Feldern rückgekoppelter Natur-Gesellschafts-Systeme zu sein, die auch als «Mensch-Umwelt-Forschung» bezeichnet werden. Die Erzeugung von Komplexität bzw. die Komplexitätszunahme derartiger Systeme erfordert erweiterte und neue Zugänge zu den theoretischen, methodischen und empirischen Grundlagen der Disziplin. Die Komplexitätstheorie wird daher ein wichtiges wissenschaftliches Entwicklungsfeld der zukünftigen geomorphologischen Forschung darstellen müssen. $\mathrm{Zu}$ einigen Punkten dieser perspektivischen Themenstellung wird der Deutsche Arbeitskreis für Geomorphologie in diesem Jahr eine Denkschrift herausgeben (DeuTscher ArbertsKREIS FÜR GEOMORPHOLOGIE 2006).

Was unter den Gesichtspunkten der geomorphologischen Theorieentwicklung nun ansteht, ist eine Verknüpfung der Theorie des nichtlinearen, komplexen Systemverhaltens mit der multiskaligen, mehrphasig-polygenetischen Theorie der Erdoberfläche. Erst dadurch kann eine Basis geschaffen werden für weitergehende Fragenstellungen, wie z.B.

- das «Durchreichen» zyklischen Verhaltens externer Einflussvariablen, z.B. des Klimas, auf mehrere subskalige Ebenen geomorphologischer Systeme,

- die Evolution der mehrphasig-polygenetischen Komponenten des Erdoberflächensystems als Folge systeminterner Organisationsprozesse,

- der zeitliche Wechsel von Zuständen des Systemgleichgewichtes und -nichtgleichgewichtes in gleicher Raumskale,

- die Rückkopplung emergenter Eigenschaften von Systemen auf unterschiedlichen Raumskalen durch zyklische oder episodische Einflüsse variabler Raumskalen oder

- die Komplexitätserzeugung bzw. Reduktion von Resilienz («resilience reduction») durch externe Störungen, z.B. durch die menschliche Kolonisierung.

Diesen Fragen konnte in diesem Aufsatz nicht nachgegangen werden. Ohne Zweifel bilden sie im Rahmen der existierenden Paradigmen der Geomorphologie massive Herausforderungen nicht nur an Semantik, Theorie und Konzeption, sondern auch an die wissenschaftssoziologischen Prozesse innerhalb der Disziplin. Diese Herausforderungen müssen jedoch angenommen werden, um nicht den Anschluss an die Wissenschaften des Systems Erde («earth system sciences») zu verlieren (STEFFEN et al. 2004), und um für die «Mensch-Umwelt-Forschung» gerüstet zu sein.

Innovationen, mit denen die geomorphologische Disziplin heute konfrontiert ist, umfassen in starkem Maße auch neue Verfahrenstechniken und Methoden. Sie stellen einen Schwerpunkt dieses Bandes der Geographica Helvetica dar. Komplementär zu diesen Beiträgen soll im Folgenden auf einige Verfahren verwiesen werden, mit denen in der Bonner Arbeitsgruppe einige Erfahrungen gesammelt werden konnten, und die im Rahmen des Graduiertenkollegs 437 (Das Relief - eine strukturierte und veränderliche Grenzfläche) eingesetzt werden (http://www.giub.unibonn.de/grk/). 
Die neuen und verbesserten Techniken der Datenerhebung gewähren einen deutlich verbesserten Einblick in den geomorphologisch relevanten Untergrund durch geophysikalische Sondierung (SCHROTT et al. 2003) und weiterentwickelte Datierungsverfahren für Fest- und Lockergestein (Summerfield 2005). Ein Problem bereitet jedoch oftmals die Messung und Quantifizierung des Massenversatzes der Erdoberfläche, die über Punktmessungen hinausgehen, die gesamte Fläche des Objektes betreffen und in die räumliche Mesoskale vorstoßen. Die Verfügbarkeit von digitalen Höhendaten aus flugzeuggestützten oder terrestrischen Laserscan-Verfahren hat hier deutliche Fortschritte gebracht. Darüber hinaus sollte, so ein Vorschlag, zukünftig erwogen werden, die folgenden Technologien verstärkt in der geomorphologischen Geländeerkundung zu nutzen:

- Differentielle SAR-Interferometrie zur Detektion der Kinematik der Erdoberfläche (VIETMEIER 1998)

- Digitale Photogrammetrie zur Detektion der Kinematik von Gletschern und Blockgletschern (ROER et al. 2005a, 2005b; Roer 2005)

- High Resolution Stereo Camera (HRSC) (digitale Höhendaten, schwarzweiße und multispektrale Luftbilder) für die Detektion von Sedimentspeichern und die Erdoberflächenkinematik (NYENHUIS et al. 2005; Nyenhuis 2005; Roer 2005).

Ein besonderes Anliegen ist die Nutzung der geomorphologischen Kartographie im Sinne der GMK25/ GMK100-Konzeption (BARSCH \& LiedTKE 1980) und ihre digitale Weiterentwicklung (DIKAU \& SCHMIDT 1999; OtTo \& Dikau 2004; Rasemann 2004; Rasemann et al. 2004). Die Potenziale der wissenschaftlichen Kartographie für die geomorphologische Disziplin können nicht hoch genug eingeschätzt werden.

Neue technologische Entwicklungen bieten nicht nur die Gelegenheit, durch neue Daten «alte» Theorien zu verifizieren, wie dies durch Summerfield (2005) für die geomorphogenetische Rekonstruktion und Modellierung ausgeführt wurde. Sie könnten auch dazu verleiten, den epistemologischen Diskurs in der Disziplin zu vernachlässigen, weil ja nun den empirischen Bedürfnissen des Forschers ausreichend gedient ist und das «hunting for data» erfolgreich war. Gerade durch Erfahrungen in der Erforschung von NaturGesellschafts-Wechselwirkungen (MÜLLER-MAHN \& WARDENGA 2005) sei vor derartigen Kurzschlüssen gewarnt. Auch neue Technologien entbinden nicht von der Notwendigkeit, die paradigmatischen Entwicklungen der Geomorphologie und ihrer Nachbardisziplinen zu kennen und in den Kontext der gesellschaftlichen Relevanz der Wissenschaften zu stellen. Diese Kenntnisse bilden ein sine qua non für eine erfolgreiche Weiterentwicklung der Geomorphologie durch die jetzt junge Forschergeneration.
Die dem Autor vorgegebene und im Titel dieses Aufsatzes gestellte Frage kann also wie folgt beantwortet werden:

\section{«Oberflächenprozesse»: nicht nur}

Die Erdoberfläche ist und bleibt das Zentrum der geomorphologischen Forschung und Lehre. Es ist die sich verändernde Oberfläche des Erdkörpers und Grenzfläche zu anderen Komponenten des Systems Erde. Die Erdoberfläche und die sie verändernden Prozesse können nur als Einheit aufgefasst werden. Sie bilden den Lebensraum des Menschen.

«Mit der Erklärung einer so umfassenden, für alles Leben und den Menschen selbst so bedeutsamen Naturerscheinung, wie sie eben die Reliefsphäre darstellt, nimmt die Geomorphologie einen selbständigen und notwendigen Platz im System der Naturwissenschaften ein, den keine andere ausfüllen kann» (BüDEL 1981: 13).

«ein altes Thema?»: ja

«Wer nur das Wissen seiner Generation kennt, bleibt selbst ein Kind» (übersetzte Inschrift an der Hauptbibliothek der University of Colorado, Boulder, USA).

«ein neues Thema?»: ja

"Complexity is itself a complex notion that combines compositional, structural, and functional elements» (RESCHER 1998: xiii).

«Many of our resource and environmental problems involve the complexity of interactions between natural and social systems» (BERKES et al. 2003:7).

\section{Literatur}

BARSCH, D. (1981): Studien zur gegenwärtigen Geomorphodynamik im Bereich der Oobloyah Bay, NEllesmere Island, N.W.T., Kanada. - In: BARSCH, D. \& L. KING (Hrsg.): Ergebnisse der Heidelberg-Ellesmere Island-Expedition. - In: Heidelberger geographische Arbeiten 69: 123-161.

Barsch, D. \& H. LiedtKe (1980): Principles, scientific value and practical applicability of the geomorphological map of the Federal Republic of Germany at the scale of 1:25,000 (GMK 25) and 1:100,000 (GMK 100). - In: Zeitschrfit für Geomorphologie, N.F., Supplementband 36: 296-313.

BARSCH, D. \& N.T. CAINE (1984): The nature of mountain geomorphology. - In: Mountain research and development 4, 4: 287-298.

Bauer, B.O., Veblen, T.T. \& J.A. Winkler (1999): Old methodological sneakers: fashion and function in a cross-training era. - In: Annals of the Association of American Geographers 89: 679-687.

BECKER, E. \& T.JAHN (2006): Soziale Ökologie - Grund- 
züge einer Wissenschaft von den gesellschaftlichen Naturverhältnissen. - Frankfurt am Main: Campus.

Berkes, F., Colding, J. \& C. Folke (2003): Navigating social-ecological systems. - Cambridge: Cambridge University Press.

Beven, K. (1996): Equifinality and uncertainty in geomorphological modelling. - In: RHOADS, B.L. \& C.E. THORN (eds): The scientific nature of geomorphology. Proceedings of the $27^{\text {th }}$ Binghamton Symposium in Geomorphology held 27-29 September 1996. - Chichester: Wiley: 289-313.

Bremer, H. (1989): Allgemeine Geomorphologie. - Berlin: Gebrüder Borntraeger.

Brown, A. G. \& T.A. Quine (eds) (1999): Fluvial processes and environmental change. - Chichester: Wiley. BRUNSDEN, D. (1996): Geomorphological events and landform change. - In: Zeitschrift für Geomorphologie, N.F., 40: 273-288.

BüDEL, J. (1981) Klima-Geomorphologie. - Berlin: Gebrüder Borntraeger.

Chorley, R., Schumm, S.A. \& D.E. Sugden (1984): Geomorphology. - London: Methuen.

DeARING, J.A. \& R.T. Jones (2003): Coupling temporal and spatial dimensions of global sediment flux through lake and marine sediment records. - In: Global and planetary change 39: 147-168.

Dearing, J.A., Battarbee, R.W., Dikau, R., Larocque, I. \& F. OldFIELD (2006a): Human-environment interactions: learning from the past. - In: Regional environmental change 6:1-16.

Dearing, J.A., Battarbee, R.W., Dikau, R., LarocQUE, I. \& F. OldField (2006b): Human-environment interactions: towards synthesis and simulation. - In: Regional environmental change 6:115-123.

Deutscher Arbeitskreis für GeOMORPhOlogie (Hrsg.) (2006): Die Erdoberfläche - Lebens- und Gestaltungsraum des Menschen. - In: Zeitschrift für Geomorphologie, N.F., Supplementband (im Druck).

DiKAU, R. (1994): Computergestützte Geomorphographie und ihre Anwendung in der Regionalisierung des Reliefs. - In: Petermanns geographische Mitteilungen 138: 99-114.

DiKAU, R. (1999): The need for field evidence in modelling landform evolution. - In: Hergarten, S. \& H. Neugebauer (eds): Process modelling and landform evolution. - In: Lecture notes in earth sciences 78, Heidelberg: 3-12.

DiKAU, R. (2004): Die Bewertung von Naturgefahren als Aufgabenfeld der Angewandten Geomorphologie. - In: Zeitschrift für Geomorphologie, N.F., Supplementband 136: 181-193.

DiKaU, R. (2005): Geomorphologische Perspektiven integrativer Forschungsansätze in Physischer Geographie und Humangeographie. - In: Müller-MaHN, D. \& U. WARDENGA (Hrsg.): Möglichkeiten und Grenzen integrativer Forschungsansätze in Physischer Geographie und Humangeographie. - In: Forum IFL 2, Leipzig: 91-108.
DIKAU, R. \& J. SCHMIDT (1999): Georeliefklassifikation. - In: Schneider-Sliwa, R., Schaub, D. \& G. Gerold (Hrsg.): Angewandte Landschaftsökologie - Grundlagen und Methoden. - Heidelberg: Springer: 217-244.

Dikau, R., Herget, J. \& K. Hennrich (2005): Land use and climate impacts on fluvial systems during the period of agriculture in the River Rhine catchment (RhineLUCIFS) - an introduction. - In: Erdkunde 59: 177-183.

Dix, A. \& K.-D. KLeEfeld (2005): Kulturlandschaftswandel und Landnutzung als «human impacts» - Methodische Teilergebnisse des DFG-Projektes RheinLUCIFS. - In: Ebert, J., BaIerL, C. \& I. MarSCHALL (Hrsg.): Landwirtschaftliche Großbetriebe und Landschaft im Wandel. Die hessische Domäne Frankenhausen im regionalen Vergleich (16. bis 20. Jahrhundert). - Bielefeld: Verlag für Regionalgeschichte: 253-282.

Favis-Mortlock, D. (2004): Self-organization and cellular automata models. - In: WAINWRIGHT, J. \& M. Mulligan (eds): Environmental modelling. Finding simplicity in complexity. - Chichester: Wiley: 349-369. Gregory, K.J., Benito, G., Dikau, R., Golosov, V., Jones, A.J.J., Macklin, M.G., Parsons, A.J., Passmore, D.G., Poesen, J., Soja, R., Starkel, L., Thorndycraft, V.R. \& D.E. WALLING (2006): Past hydrological events to understanding global change: An ICSU research project. - In: Catena 66: 2-13.

Harrison, S. (2001): On reductionism and emergence in geomorphology. - In: Transactions of the Institute of British Geographers 26, 3: 327-339.

HERGET, J. \& R. DikAu (eds) (2005): Natural and human impacts in the River Rhine catchment. - In: Erdkunde 59,3/4 (special issue), Bonn.

Hoffmann, T., Erkens, G., Dikau, R., Houben, P. \& J. SEIDEL (2006): Holocene sediment storage and erosion within the Rhine catchment. - In: The Holocene (in press).

Houben, P., Hoffmann, T., Zimmermann, A. \& R. DIKAU (2006): Land use and climatic impacts on the Rhine system during the period of agriculture (RheinLUCIFS). - In: Catena 66: 42-52.

KIRKBY, M.J. (1996): A role for theoretical models in geomorphology. - In: RHOADs, B.L. \& C.E. THORN (eds): The scientific nature of geomorphology. Proceedings of the $27^{\text {th }}$ Binghamton Symposium in Geomorphology held 27-29 September 1996.- Chichester: Wiley: 257-272.

KIRKBy, M.J. (1999): Landscape modelling at regional to continental scales. - In: Hergarten, S. \& H.J. Neugebauer (eds): Process modelling and landform evolution. - Lecture notes in earth sciences 78 , Berlin: Springer: 189-203.

Kugler, H. (1974) Das Georelief und seine kartographische Modellierung. - Dissertation B, MartinLuther-Universität Halle-Wittenberg, Fakultät für Naturwissenschaften. 
Kugler, H. \& D. Schaub (1997): Allgemeine Geomorphologie. - In: HendL, M. \& H. LiedTKE (Hrsg.): Lehrbuch der allgemeinen physischen Geographie. - 3., überarbeitete und erweiterte Auflage, Gotha: Perthes: 141-231.

LANE, S.N. \& K.S. Richards (1997): Linking river channel form and process: time, space and causality revisited. - In: Earth surface processes and landforms 22: 249-260.

MAINZER, K. (2004): Thinking in complexity. - Heidelberg: Springer.

MCCANN, S.B. \& D.C. Ford (1996): Geomorphology sans frontières. - Chichester: Wiley.

Müller-MahN, D. \& U. Wardenga (Hrsg.) (2005): Möglichkeiten und Grenzen integrativer Forschungsansätze in Physischer Geographie und Humangeographie. - = Forum IFL 2, Leipzig.

Nyenhuis, M. (2005): Permafrost und Sedimenthaushalt in einem alpinen Geosystem. - Dissertation am Geographischen Institut der Universität Bonn, http:// hss.ulb.uni-bonn.de/diss_online/math_nat_fak/2005/ nyenhuis_michael/ 29.12.2005.

Nyenhuis, M., Hoelzle, M. \& R. Dikau (2005): Rock glacier mapping and permafrost distribution modelling in the Turtmanntal, Valais, Switzerland. - In: Zeitschrift für Geomorphologie, N.F., 49: 275-292.

Отто, J. \& R. DiKaU (2004): Geomorphologic system analysis of a high mountain valley in the Swiss Alps. In: Zeitschrift für Geomorphologie, N.F., 48: 323-341.

PASSARge, S. (1912): Physiologische Morphologie. - In: Mitteilungen der Geographischen Gesellschaft Hamburg 26: $135-337$.

PENCK, A. (1894): Morphologie der Erdoberfläche. - Stuttgart: Engelhorn.

Phillips, J.D. (1992): The end of equilibrium? - In: Geomorphology 5: 195-201.

PhILlips, J.D. (2003): Sources of nonlinearity and complexity in geomorphic systems. - In: Progress in physical geography 27: 1-23.

Prigogine, I. (1992): Vom Sein zum Werden - Zeit und Komplexität in den Naturwissenschaften. - 6., überarbeitete und erweiterte Auflage, München, Zürich: Piper.

Prigogine, I. (1997): The end of certainty - Time, chaos, and the new laws of nature. - New York: Free Press.

Rasemann, S., Schmidt, J., Schrott, L. \& R. Dikau (2004): Geomorphometry in mountain terrain. - In: Bishop, M. \& J.F. Shroder (eds): Geographic information science in mountain geomorphology. - Berlin: Springer: 101-145.

RASEMANN, S. (2004): Geomorphometrische Struktur eines mesoskaligen alpinen Geosystems. - In: Bonner geographische Abhandlungen 111, Bonn, http://hss. ulb.uni-bonn.de/diss_online/math_nat_fak/2003/rasemann_stefan/index.htm 15.3.2006.

Rescher, N. (1998): Complexity. A philosophical overview. - New Brunswick: Transaction Publishers.
Rhoads, B.L. \& C.E. Thorn (1996): The scientific nature of geomorphology. - Chichester: Wiley.

RICHARDS, K.S. (2002): Drainage basin structure, sediment delivery and the response to environmental change. - In: Jones, S.J. \& L.E. Frostrck (eds): Sediment flux to basins: causes, controls and consequences, - London: The Geological Society of London, Special Publication: 149-160.

Richards, K., Arnold, N., Lane, S., Chandra, S., ElHames, A. \& N. MatTikalli (1995): Numerical landscapes: static, kinematic and dynamic process-form relations. - In: PIKE, R. \& R. DikAU (eds): Advances in geomorphometry. Zeitschrift für Geomorphologie, N.F., Supplementband 101, Stuttgart: 201-220.

RoER, I. (2005): Rockglacier kinematics in a high mountain geosystem. - Dissertation am Geographischen Institut der Universität Bonn, http://hss.ulb. uni-bonn.de/diss_online/math_nat_fak/2005/roer_isabelle/index.htm 28.12.205.

Roer, I., KäÄв, A. \& R. Dikau (2005a): Rockglacier acceleration in the Turtmann valley (Swiss Alps): Probable controls. - In: Norsk geografisk tidsskrift 59:157163.

Roer, I., KäÄB, A. \& R. DiKaU (2005b): Rockglacier kinematics derived from small-scale aerial photography and digital airborne pushbroom imagery. - In: Zeitschrift für Geomorphologie, N.F., 49: 73-87.

Rohdenburg, H. (1989): Landschaftsökologie - Geomorphologie. - Cremlingen-Destedt: Catena.

Schrott, L., Hördt, A. \& R. Dikau (Hrsg.) (2003): Geophysical applications in geomorphology. - = Zeitschrift für Geomorphologie, Supplementband 132, Stuttgart.

Schumm, S.A. (1973): Geomorphic thresholds and complex response of drainage systems. - In: MoRISAWA, M. (ed.): Fluvial geomorphology: Proceedings of the Fourth Annual Geomorphology Symposium held at Binghamton, September 27-28, 1973: 299-310.

Schumm, S.A. (1979): Geomorphic thresholds: the concept and its applications. - In: Transactions of the Institute of British Geographers, NS 4: 485-515.

Schumm, S.A. \& R.W. Lichty (1965): Time, space and causality in geomorphology. - American Journal of Science 263: 110-119.

Schumm, S.A. (1991):To interpret the earth - ten ways to be wrong. - Cambridge: Cambridge University Press.

SlaymaKer, O. (1997): A pluralist, problem-focused geomorphology. - In: StodDart, D.R. (ed.): Process and form in geomorphology. - London, New York: Routledge: 328-339.

SPEDDING, N. (1997): On growth and form in geomorphology. - In: Earth surface processes and landforms 22: 262-265.

Stephan, A. (1999): Emergenz - Von der Unvorhersagbarkeit zur Selbstorganisation. - Dresden: Dresden University Press.

Steffen, W., Sanderson, A., Jäger, J., Tyson, P.D., 
Moore III, B., Matson, P.A., Richardson, K., OldField, F., SChellnhuber, H-J., TuRner II, B.L. \& R.J. WASSON (2004) (eds): Global change and the earth system: a planet under pressure. -IGBP Book Series, Heidelberg: Springer.

Sugden, D.E. (1996): The East Antarctic Ice Sheet: unstable ice or unstable ideas? - In: Transactions of the Institute of British Geographers, NS 21:443-454.

Sugden, D.E., Summerfield, M.A. \& T.P. Burt (1997): Editorial: Linking short-term geomorphic processes to landscape evolution. - In: Earth surface processes and landforms 22: 193-194.

Summerfield, M.A. (2000): Geomorphology and global tectonics: Introduction. - In: SUMMERFIELD, M.A (ed.): Geomorphology and global tectonics. - Chichester: Wiley: 3-12.

Summerfield, M.A. (2005): The changing landscape of geomorphology. - In: Earth surface processes and landforms 30: 779-781.

TRIMBLE, S.W. (1999): Decreased rates of alluvial sediment storage in the Coon Creek Basin, Wisconsin, 1975-93. - In: Science 285: 1244-1246.

VieTMEIER, J. (1998): Die differentielle SAR-Interferometrie zur Untersuchung von Massenbewegungen. Unveröffentlichte Diplomarbeit am Geographischen Institut der Universität Bonn.

WASSON, R.J. (2002): Sediment budgets, dynamics, and variability: new approaches and techniques. - In: Dyer, F.J., Thoms, M.C. \& J.M. Olley (eds): The structure, function and management implications of fluvial sedimentary systems. - = IAHS Publication No. 276, Wallingford: 471-478.

Zimmermann, A., Richter, J., Frank, T. \& K.P. Wendt (2004): Landschaftsarchäologie II - Überlegungen zu Prinzipien einer Landschaftsarchäologie. - = Bericht der Römisch-Germanischen Kommission 85, Mainz: 37-95.

\section{Zusammenfassung: Oberflächenprozesse - ein altes oder ein neues Thema?}

Der vorliegende Aufsatz thematisiert aktuelle Diskurse über die geomorphologischen Beiträge zu sich verändernden Forschungszielen und Paradigmen innerhalb der Erdsystem-Wissenschaften. Auf Basis des überzeugenden Paradigmas der Reliefgenerationen als multiskaliges Palimpsest von Reliefformen sollte die zukünftige geomorphologische Forschung auf den folgenden Feldern vorangetrieben werden: (1) raum-zeitskalen-bezogene Methoden, (2) Kopplung neuer Ansätze der tektonischen Modellierung und Datierungstechnologien mit einem geomorphologischem Verständnis der Geomorphogenese, (3) nichtlineare Komplexität in geomorphologischen Systemen und (4) Sedimenthaushalte in biogeochemischen Kreisläufen. Es erscheint jedoch notwendig darauf hinzuweisen, dass neue Technologien nicht die epistemologische Debatte in der Disziplin ersetzen können.

\section{Summary: Land surface processes - an old or new issue?}

This paper is embedded within the ongoing discourse on the role of geomorphology within the changing field of the earth system sciences. It argues that based on the strong paradigm of multi-scaled landform palimpsests, geomorphic research should further its activity within the areas of (1) space/time-scale related methods, (2) coupling new approaches of tectonic modelling and dating technologies with geomorphic understanding of landscape evolution, (3) non-linear complexity in geomorphic systems, and (4) sediment budgets within biogeochemical cycles. However, it is emphasised that the orientation towards new technologies does not tangent on the epistemological debate within the discipline.

\section{Résumé: Les processus de surface: un thème d'actualité?}

Cet article traite des débats contemporains relatifs à la contribution de la géomorphologie aux objectifs et paradigmes des sciences de la terre. Il postule que les recherches géomorphologiques devraient s'appuyer sur une analyse multiscalaire des formes du relief de manière à promouvoir quatre champs de recherche: (1) les méthodes traitant de l'espace et du temps, (2) le couplage des nouvelles approches de modélisation tectonique et de techniques de datation avec la compréhension géomorphologique de l'évolution du paysage, (3) la complexité non linéaire des systèmes géomorphologiques et (4) les budgets sédimentaires à l'intérieur des cycles biogéochimiques. Cependant, il semble nécessaire de relever le fait que les nouvelles technologies ne peuvent remplacer le débat épistémologique dans la discipline.

Prof. Dr. Richard Dikau, Geographisches Institut der Universität Bonn, Meckenheimer Allee 166, D-53115 Bonn, Deutschland.

e-mail: rdikau@giub.uni-bonn.de

\section{Manuskripteingang/received/manuscrit entré le \\ 13.1.2006}

Annahme zum Druck/accepted for publication/accepté pour l'impression: 14.9.2006 